\author{
V. L. Markine • I. Y. Shevtsov • C. Esveld
}

\title{
An inverse shape design method for railway wheel profiles
}

Received: 1 April 2006 / Published online: 28 September 2006

(C) Springer-Verlag 2006 ticated solutions have been introduced in recent years on the railways. Despite of this progress, the mechanics of a railway wheelset remains unchanged, and an inappropriate combination of wheel and rail profiles can easily diminish all these technological advances. Besides, many old-fashioned vehicles are still in too good condition to be replaced. They have a special need for appropriate combinations of wheel/rail profiles because such vehicles do not have high-tech devices that improve their performance.

Design of a wheel profile is an old problem, and various approaches were developed to obtain a satisfactory combination of wheel and rail profiles. Usually, it is possible to find an optimal combination of wheel and rail profiles when dealing with a closed railway system, i.e., when only one type of rolling stock is running on a track and no influence of other types of railway vehicles is present. Examples of such systems are heavy haul and tramlines. In the present paper, a closed railway system is considered, namely, the metro network in Rotterdam (RET), The Netherlands.

Due to conical shape of wheels, the centre of a wheelset axle produces sinusoidal motion, a so-called Klingel motion, when it travels along a track (Fig. 1). The kinematical properties of wheel and rail contact such as a rolling radius, contact angles and wheelset roll angle vary as a wheelset moves laterally relative to the rails. The nature of the functional dependence between these geometrically constrained variables and the wheelset lateral position is defined by the cross-sectional shape of the wheel and rail. By studying the geometrical characteristics of contact between wheel and rail, it is possible to judge about the dynamic behaviour of the corresponding wheelset and ultimately about the dynamic properties of the vehicle because the motion of the wheelset is the source of vehicle excitations.

The wheel and rail shapes define not only the kinematical and dynamical properties of a wheelset but also the physical properties of wheel and rail contact such as contact stresses, creep and wear. A wrong combination of wheel and rail profiles can be a source of various railway problems such as high wear rate of the wheels, instability (hunting) of a wheelset as well as rolling contact fatigue defects of the rails. Thus, the wrong choice of wheel and rail profiles can ultimately result in cost-inefficient (due to a short lifetime of wheels)
V. L. Markine $(\varangle) \cdot$ I. Y. Shevtsov · C. Esveld

Section of Road and Railway Engineering, Faculty of Civil Engineering and Geosciences, Delft University of Technology, Stevinweg 1

2628 CN, Delft, The Netherlands

e-mail: V.L.Markine@TUDelft.NL

e-mail: I.Y.Shevtsov@TUDelft.NL

e-mail: C.Esveld@TUDelft.NL 


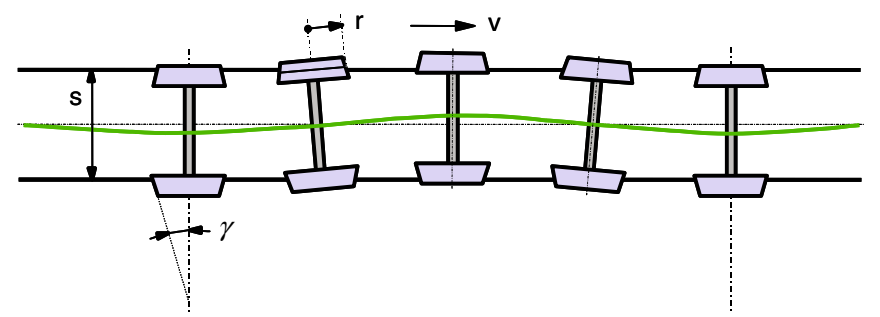

Fig. 1 Klingel motion of a wheelset

and unsafe (due to possible wheelset derailment) design of a railway vehicle.

Even though the problem of wheel and rail interface is very complex and various disciplines are required to analyse it (such as vehicle dynamics, contact mechanics, metallurgy, etc.), the majority of the interface problems can be solved by the proper choice of the cross-sectional shapes of the wheel and rail. Usually, rails are already present in a railway network, and it is difficult to change the shape of the rail profile. Only small changes in the cross-sectional shape of rails can be achieved by grinding. Therefore, one can try to improve the wheel and rail interface by adjusting the shape of the wheel profile.

Typically, a wheel profile was designed using the trial and error approach. The choice of the wheel cross-sectional shape was mostly based on the designer's intuition and experience as well as the measurement data. During the last decades, a number of efforts have been made to use numerical methods in the wheel design process. Smith and Kalousek (1990) developed a numerical procedure for design of a wheel profile described by a series of arcs. Although the procedure was specifically developed for steered axle vehicles, some important aspects of it can be applied to conventional systems as well. The process of designing a new wheel profile for North American railroads was described by Leary et al. (1990) wherein alternative profiles were derived through two techniques: an average worn wheel profile and profiles based on expansions of rail shapes. Both methods provided good base designs for candidate profiles. However, the initial wheel shapes produced were modified to suit the specific concerns of the task involved. This was done through careful computer analysis of the dynamic and contact stress characteristics of each wheel profile. One procedure for design of a wheel profile using a numerical optimisation technique was proposed in the works of Shevtsov et al. (2002a, b). In these papers, the suggested optimality criteria in those for wheel profile design were based on a rolling radii difference (RRD) function. In that procedure, the optimisation searches for an optimum wheel profile by minimising the difference between the target (desired) and actual RRD functions. To solve the minimisation problem, an optimisation method that uses multipoint approximations based on response surface fitting (MARS) method was used. In the studies of Shevtsov et al. (2003, 2005), the procedure was further developed, and three approaches for choosing the target RRD function were suggested. Similar approach was proposed in Shen et al. (2003) wherein the contact angle function was used for design of railway wheel profiles. Using an inverse method for known contact angles and rail profile, a corresponding wheel profile was found. Persson and Iwnicki (2004) used a direct optimisation procedure based on a genetic algorithm for design of a wheel profile for railway vehicles. Two existing wheel profiles were chosen as parents, and genes were formed to represent these profiles. These genes were mated to produce offspring genes and then reconstructed into profiles that had random combinations of the properties of the parents. Each of the offspring profiles was evaluated by running a computer simulation of the vehicle behaviour and calculating a penalty index. The inverted penalty index was used as the fitness value in the genetic algorithm. The method was used to obtain optimised wheel profiles for two vehicles, one with relatively soft primary suspensions and the other one with relatively stiff primary suspensions.

In this paper, a procedure described in the studies of Shevtsov et al. $(2003,2005)$ is applied to improve the performance of metro vehicles that were suffering from severe wheel wear and instability (Markine et al. 2004). The RRD function used in the formulation of the optimality criteria is described in the Section 2. The proposed wheel design procedure and the optimisation method are described in the Section 3 and the Section 4 respectively. The results of the optimisation of a wheel profile are presented and discussed in the Section 5.

\section{Wheel-rail contact properties}

One important characteristic of contact between wheel and rail is the rolling radius of a wheel at the contact point (Dukkipati 2000). In fact, that radius can be different for the right and the left wheels as a wheelset is moving along a track (radii $r_{1}$ and $r_{2}$, respectively, in Fig. 2).

When a wheelset is in a central position, the rolling radii of the left and the right wheels are the same, namely, $r_{1}=$ $r_{2}=r$. An instantaneous difference between the rolling radius of the right and the left wheels can be defined as a func-

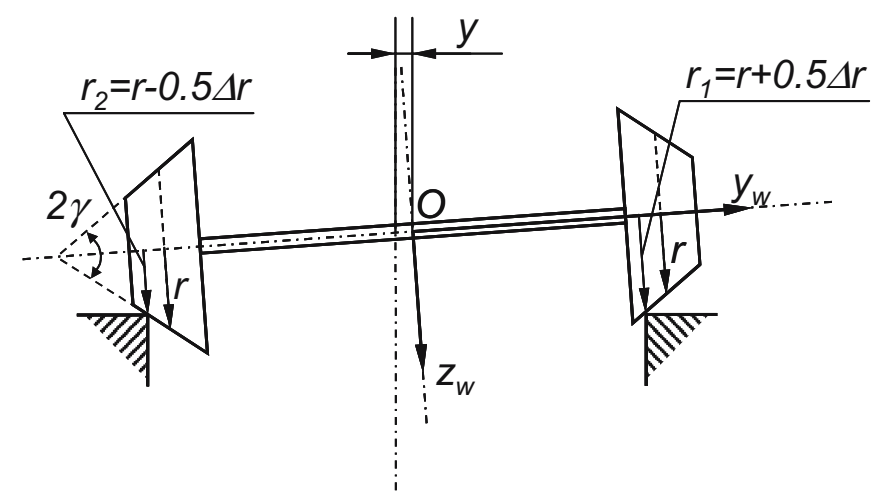

Fig. 2 Rolling radii $\left(r_{1}\right.$ and $\left.r_{2}\right)$ corresponding to wheelset displacement $y$, the wheels are conical, $\gamma$ is conicity of the wheels. The wheelset coordinate system is $y_{w} O z_{w}$ 


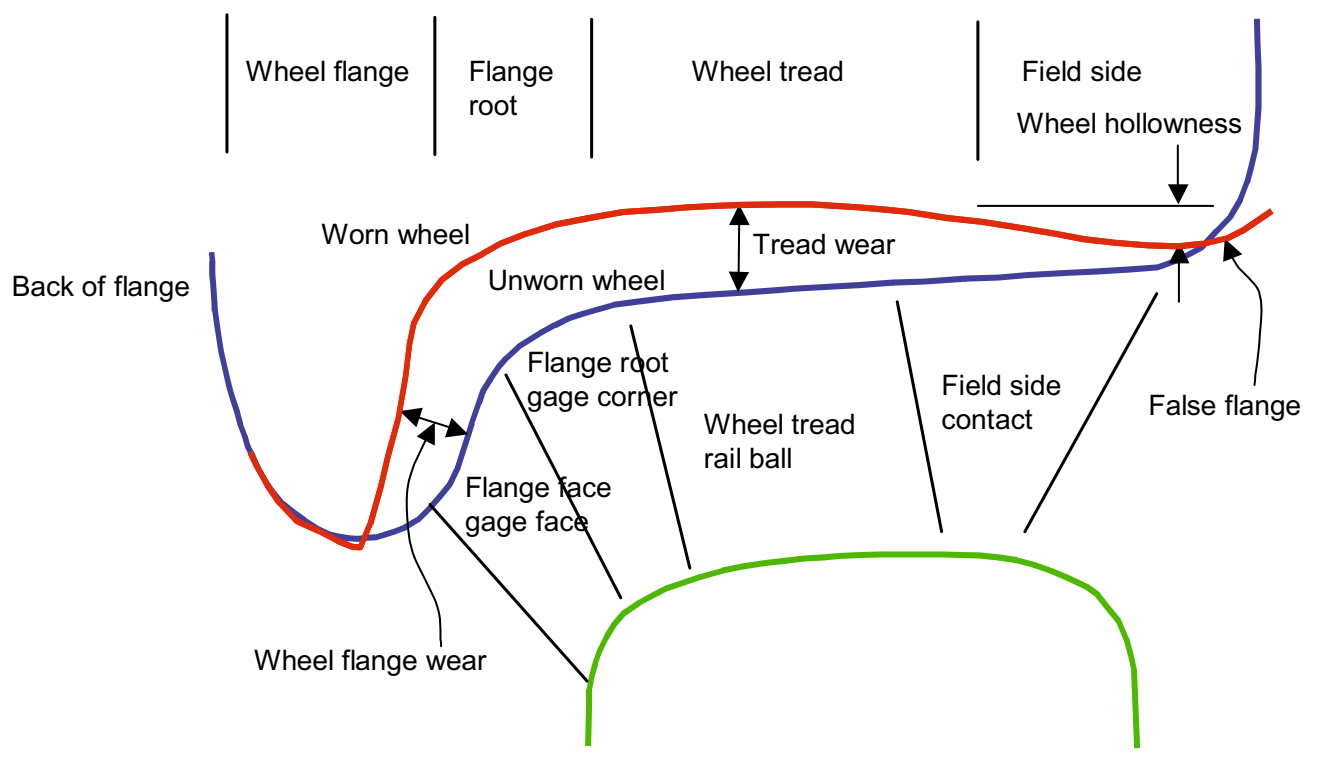

Fig. 3 Worn and unworn wheels

tion of the lateral displacement $y$ of a wheelset with respect to its central position (Fig. 2) that reads:

$\Delta r(y) \equiv r_{1}(y)-r_{2}(y)$

The RRD defines to a large extent the behaviour of a wheelset on a track. For example, to pass a sharp curved track without slippage between wheels and rails, larger RRD of a wheelset is needed. Avoiding slippage is important from wheel and rail wear point of view.

Generally, the RRD is a non-linear function of the lateral displacement $y$ of a wheelset, which from geometrical considerations (Fig. 2) can be written as:

$\Delta r=2 y \gamma_{e}(y)$

where $\gamma_{e}$ is the effective conicity. It should be noted that for a purely conical wheel, the effective conicity is equal to the cone angle of the wheel.

Due to wear, a wheel profile is changing (Fig. 3), and consequently, its RRD function $\Delta \mathrm{r}$ is changing as well. Examples of RRD functions (also known as a ' $y$ - $\Delta r$ ' curve) for purely conical and worn profiles are given in Fig. 4.

The equations of motion of a wheelset can be written as follows (Dukkipati 2000):

$\frac{2 f}{\mathrm{~V}} \dot{y}+k y+2 f \theta=0$

$\frac{2 f b^{2}}{V} \dot{\theta}-\frac{2 f \gamma_{\mathrm{e}} b}{r} y+c \theta=0$

where

$f \quad$ is the creep coefficient (lateral and longitudinal are equal),

$b \quad$ is the half of the transverse distance between wheelrail contact points, $\theta$ is the yaw angle,

$\gamma_{e}$ is the effective conicity,

$k$ and $c$ are the lateral and yaw stiffness of a wheelset, respectively, and

$V \quad$ is the velocity of a wheelset.

Keeping in mind (2), one can see that the difference between the rolling radii of the left and right wheels $\Delta r$ is present in the equations of motion (3). Thus, RRD is important for the dynamic behaviour of a wheelset as well (Dukkipati 2000, Esveld 2001). For example, on straight tracks when high (critical) speed of a vehicle is desired, the RRD (or equivalent conicity) should be lower to prevent instability of a wheelset.

Determination of geometrical contact characteristics for given wheel and rail profiles, wheel and rail gauge, and rail-

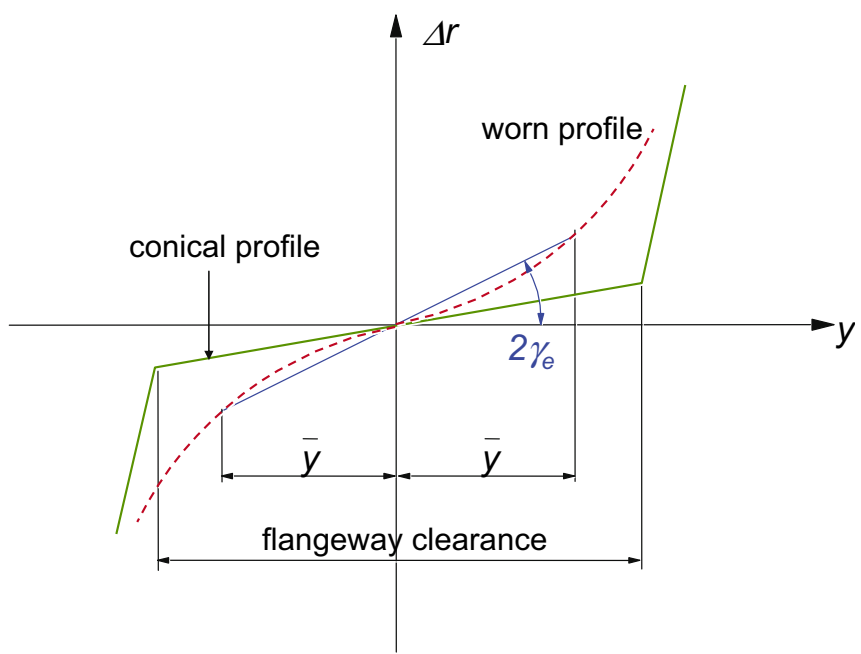

Fig. 4 Rolling radii difference vs lateral displacement of wheelset (' $y$ - $\Delta r$ ' curve) 
head cant angle is a well-known problem already solved for many years. These non-linear characteristics were investigated by Wickens (1965), Cooperrider et al. (1976) and De Pater (1988). A linear conical wheel profile widely used earlier has discontinuous linear characteristics of RRD, as shown in Fig. 4, that result in shocks when the wheel flange and rail come in contact during the motion of a wheelset. A worn wheel better matches a rail and therefore it usually has smoother RRD function. However, high conicity of a worn wheel reduces the critical speed of a wheelset and results in severe oscillations (hunting) of a vehicle. Obviously, an optimum profile is a compromise between the conical and worn profiles. A traditional way to achieve such a compromise is by manually modifying the shape of a wheel profile to obtain satisfactory contact characteristics for a given rail and by performing numerous tests with the designed wheel profiles. An optimum wheel profile should then satisfy the following requirements:

- low wear rate of wheels,

- wheelset stability and

- allowable level of wheel/rail contact stresses.

This is quite time consuming and expensive way of designing a wheel profile.

A more efficient approach is to use numerical simulation and optimisation methods in the design of a wheel profile. The approach presented in this study uses optimality criteria based on the RRD function $\Delta r$. When the optimum RRD function $\Delta r(y)$ for a given rail profile is known, one can try to solve an inverse problem to find a wheel profile that has such RRD. The problem of determination of a wheel profile corresponding to a given RRD function and rail profile is formulated as an optimisation problem. Using such approach, a wheel profile with a priori defined contact characteristics can be designed. The optimisation problem formulation, solution method and numerical results are described in the subsequent sections.

\section{Wheel design procedure}

A procedure of wheel profile design used in this study is schematically shown in Fig. 5. It consists of a number of steps. The first step is the definition of an optimum (target) RRD function. In case of improvement of the existing wheel/rail interface, wheel and rail profile measurements can be used to analyse the wheelset contact properties to design a target RRD function. Three ways of defining a target RRD function are presented and discussed in the papers of Shevtsov et al (2002a, b, 2003). An alternative approach for designing of a target RRD function is demonstrated in this study in the numerical example. Such an approach can be used in the design of a wheel profile for situations when one of the components of the existing and well-functioning vehicle/track system has been changed (new situation). For example, this is the case when new rails of a different cross-sectional shape are introduced in the existing railway network. In this case, the RRD of the wheel and rail combination from the old situation (with old rails) can be taken as the target (optimum) RRD function in the optimum design procedure of a wheel profile (Fig. 5).

In the next step of the procedure, an inverse shape design problem formulated as an optimisation problem has to be solved resulting in a new wheel profile. Because the dynamic properties of a vehicle are not directly controlled during the optimisation process, which in fact reduces the computational efforts of the optimisation, they must be verified afterwards. Thus, the dynamic performance of a vehicle with the obtained wheel profile is to be analysed to verify if the requirements on wheel/rail wear and safety have been satisfied. Here, the dynamic behaviour of a vehicle is analysed using the multibody formalism implemented in the ADAMS/Rail computer package (ADAMS/Rail 2005). If the dynamic performance of

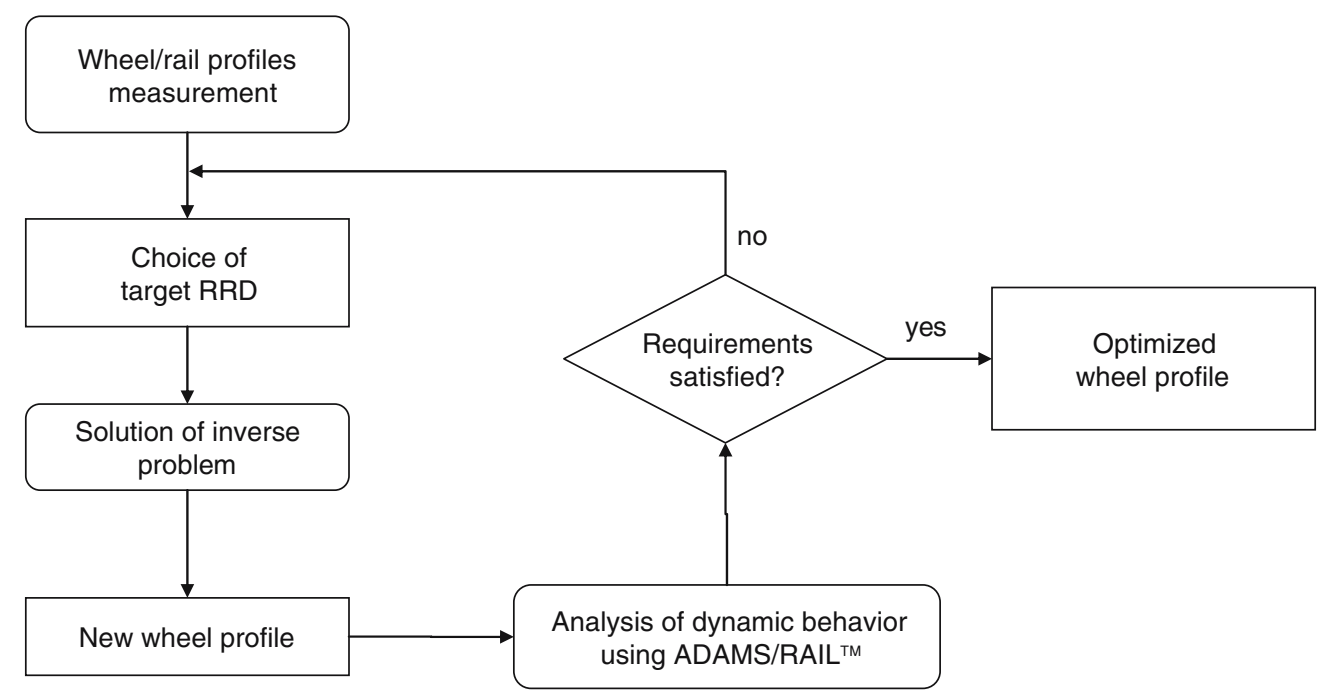

Fig. 5 Flowchart of wheel profile design procedure 
a vehicle with the obtained wheel profile does not satisfy the imposed requirements, the RRD function should be adjusted and the optimisation should be performed again. Otherwise, the obtained wheel profile can be considered as the optimum one.

\subsection{General optimisation problem}

To make use of numerical optimisation techniques, an optimisation problem should be stated in a general form that reads:

Minimise

$F_{0}(\boldsymbol{x}) \rightarrow \min , \quad \boldsymbol{x} \in R^{N}$

subject to

$F_{j}(\boldsymbol{x}) \leq 1, \quad j=1, \ldots, M$

and

$A_{i} \leq x_{i} \leq B_{i}, \quad i=1, \ldots, N$

where

$F_{0} \quad$ is the objective function;

$F_{j}, j=1, \ldots, M$ are the constraints;

$\boldsymbol{x}=\left[x_{1}, \ldots, x_{N}\right]^{T}$ is the vector of design variables and

$A_{i}$ and $B_{i}$ are the side limits, which define lower and upper bounds of the $i$-th design variable.

The components of the vector $x$ can represent various parameters of a mechanical structure, such as geometry, material, stiffness and damping properties. These properties can be varied to improve the design performance. Depending on the problem under consideration, the objective and constraint functions in (4) and (5) can describe various structural and dynamic response quantities such as weight, reaction forces, stresses, natural frequencies, displacements, velocities, accelerations, etc. Also, cost, maintenance and safety requirements can be used in the formulation of the optimisation problem. The objective function provides a basis for improvements of the design, whereas the constraints impose necessary limitations on the properties or behaviour of the structure.

Formulated in the general form (4)-(6), the optimisation problem can be solved using a conventional method of Nonlinear Mathematical Programming. Here, the MARS method has been used to solve the optimisation problem (4)-(6).

\subsection{Design variables}

To describe the geometry of a wheel profile, a number of points on its flange, flange root and tread have been chosen. Connected by a piecewise cubic Hermite interpolating polynomial, these points define the shape of the wheel profile, as shown in Fig. 6. The positions of these points can be varied to modify the profile. To reduce the computational costs of the optimisation, the positions of the points on the flange's top and on the conical part of the profile are not varied during the optimisation because these parts of the wheel profile do not participate in the wheel/rail contact.

The vertical coordinates of the other points (moving points) $z_{i}$ have been chosen as the components of the vector of the design variables $\boldsymbol{x}$ that reads:

$\boldsymbol{x}=\left[z_{1}, \ldots, z_{N}\right]$

The number of the moving points on a wheel profile and their location have been determined from the analysis of the wheel/rail contact characteristics beforehand.

\subsection{Objective function and constraints}

Because the optimum wheel profile is defined by the target RRD, the difference between its RRD and the target RRD should be as small as possible. The requirement of the minimum discrepancy between the target RRD function $\Delta r^{t a r}$ and the RRD function of the designed wheel profile $\Delta r^{\text {calc }}$ can be written as

$F_{0}(\boldsymbol{x}) \equiv \frac{\sum_{i=1}^{K}\left(\Delta r^{\text {tar }}\left(y_{i}\right)-\Delta r^{\text {calc }}\left(\boldsymbol{x}, y_{i}\right)\right)^{2}}{\sum_{i=1}^{K}\left(\Delta r^{\operatorname{tar}}\left(y_{i}\right)\right)^{2}} \rightarrow \min$,

where $y_{i}$ is the $i$-th discretisation point of the lateral displacements of a wheelset, and $K$ is the number of such points. The function (8) has been taken as the objective function of the optimisation problem (4)-(6).

Two safety requirements have been included. The first requirement is the restriction on the wheel flange thickness, which resulted in constraining the position of a design point on the wheel flange. The second one is related to prevention of vehicle derailment according to which the minimum flange angle has been prescribed. To prevent unrealistic wheel profiles, some constraints on the design variables have been imposed.

\subsection{Dynamic analysis}

When the optimisation problem has been solved, the dynamic performance of a vehicle with the obtained wheel profile

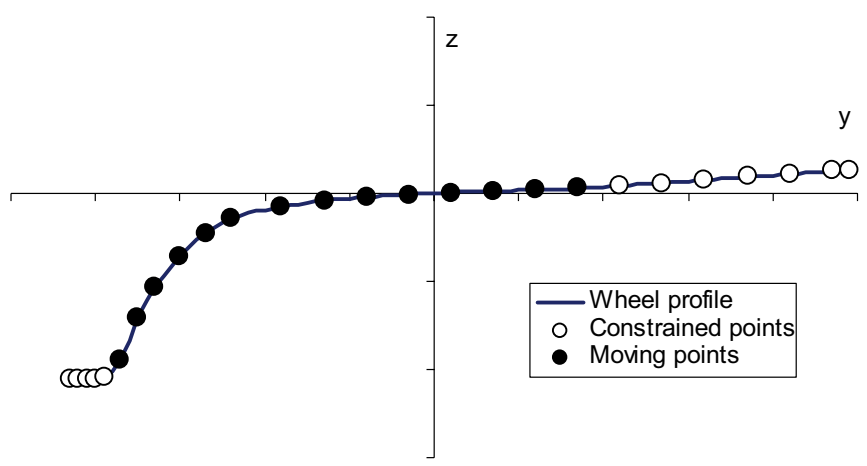

Fig. 6 Wheel profile, moving and constrained points 

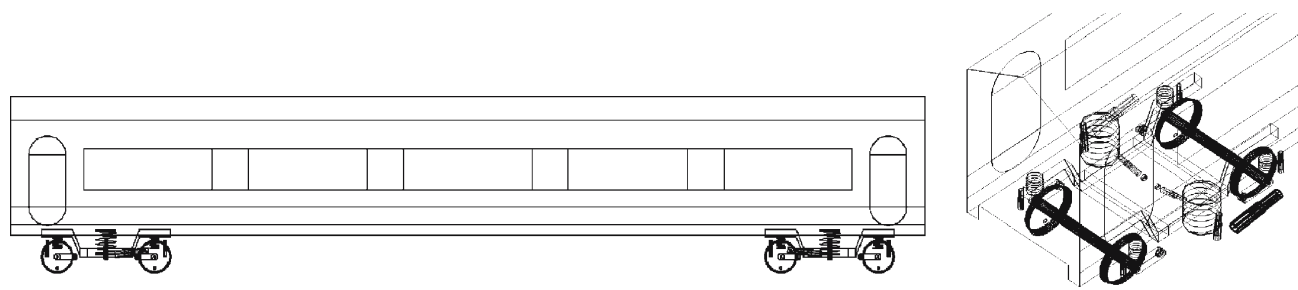

Fig. 7 ADAMS/RAIL vehicle model

should be verified. The vehicle studied here was modelled using the ADAMS/Rail computational package.

Wheel wear has been estimated using the wear index $W$, which is calculated according to the following formula (Kalker 1990):

$W=F_{1} \cdot \xi+F_{2} \cdot \eta$,

where

$F_{1}$ is the longitudinal creep force,

$\xi$ is the longitudinal creepage,

$F_{2}$ is the lateral creep force,

$\eta$ is the lateral creepage.

Wear of wheels and stability of a vehicle with the designed wheel profile on a straight track have been analysed in ADAMS/Rail (Fig. 7). The track model has the length of $700 \mathrm{~m}$, whereas at the distance of $50 \mathrm{~m}$ from the beginning of the track, a horizontal ramp (height $-5 \mathrm{~mm}$, width $-0.1 \mathrm{~m}$ ) has been introduced. The ramp induces initial disturbance to a wheelset leading to oscillations of the bogie. If the oscillations of the bogie are damping out relatively fast and their amplitude does not exceed a certain limit value, then the motion is considered as stable. The wear index has been obtained for a vehicle travelling on the same track according to (9).

It should be noted that the wheel/rail contact stresses are also available in such analysis, and they can be verified as well. However, in the numerical example presented in this study, a light rail vehicle was considered for which the contact stresses are not critical.

\section{Optimisation method}

To solve the optimisation problem formulated in the previous section, the MARS method has been used. The main features of the method are briefly described below.

\subsection{Approximation concept}

The optimisation problem in (4)-(6) can be solved using a conventional method of mathematical programming. However, when a complex system is to be analysed, the evaluations of the objective and constraint functions can be time consuming. As a result, the total computational effort of the optimisation might become prohibitive. This difficulty has been mitigated starting from the mid-70s by introducing approximation concepts (Barthelemy and Haftka 1993).

According to the approximation concepts, the original functions (4) and (5) are replaced with approximate ones, which are computationally less time consuming. Instead of the original optimisation problem (4)-(6), a succession of simpler approximated sub-problems, similar to the original one and formulated using the approximation functions, is to be solved. Each simplified problem then has the following form:

Minimise

$\widetilde{F}_{0}^{k}(\boldsymbol{x}) \rightarrow \min , \quad \boldsymbol{x} \in \boldsymbol{R}^{N}$

subject to

$\widetilde{F}_{j}^{k}(\boldsymbol{x}) \leq 1, \quad j=1, \ldots, M$

and

$A_{i}^{k} \leq x_{i} \leq B_{i}^{k}, A_{i}^{k} \geq A_{i}, B_{i}^{k} \leq B_{i}, i=1, \ldots, N$

where the superscript $k$ is the number of the iteration step, $\widetilde{F}$ is the approximation of the original function $F$, and $A_{i}^{k}$ and $B_{i}^{k}$ are move limits defining the range of applicability of the approximations.

Because the functions (10 and 11) are chosen to be simple and computationally inexpensive, any conventional method of optimisation can be used to solve the problem (10)-(12). The solution of the problem $x_{*}^{k}$ in the $k$-th iteration step is then chosen as a starting point for the next $(k+1)$-th step, and the optimisation problem (10)-(12), re-formulated with the new approximation functions $\widetilde{F}_{j}^{k+1}(x) \leqslant 1,(j=0, \ldots, M)$ and move limits $A_{i}^{k+1}$ and $B_{i}^{k+1}$, is to be solved. The process is repeated iteratively until the convergence criteria have been satisfied.

\subsection{MARS optimisation technique}

Each approximation in (10) and (11) is defined as a function of the design variables $\boldsymbol{x}$ and tuning parameters $\boldsymbol{a}$ (for brevity, the indices $k$ and $j$ will be omitted). To determine the components of vector $\boldsymbol{a}$, the following weighted least-squares mini- 


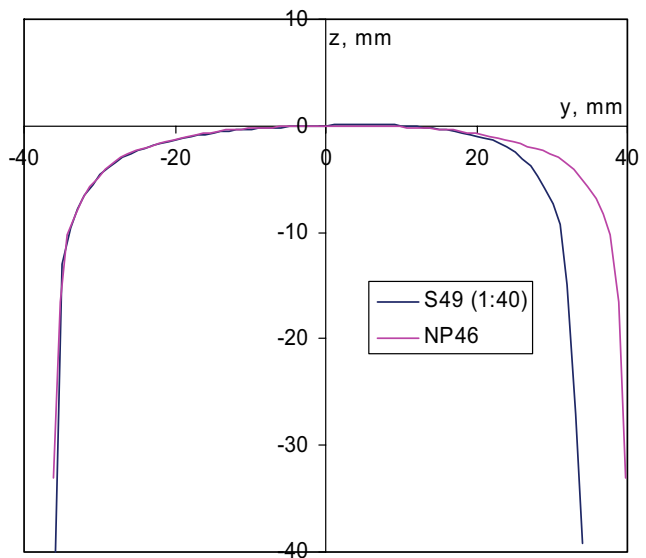

a.

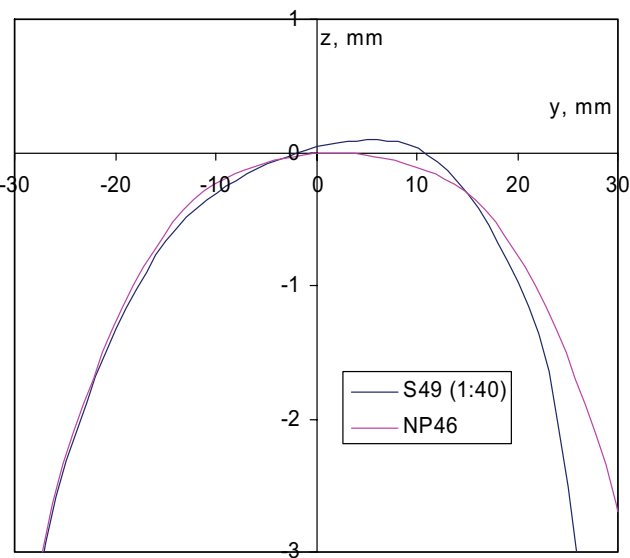

b.

Fig. 8 Comparison of rails NP46 and S49 (a not zoomed; b zoomed in)

misation problem is to be solved (Toropov 1989, Toropov and Markine 1996, Markine 1999):

Find vector $\boldsymbol{a}$ that minimises

$G(\boldsymbol{a})=\sum_{p=1}^{P}\left\{w_{p}\left[F\left(\boldsymbol{x}_{p}\right)-\widetilde{F}\left(\boldsymbol{x}_{p}, \boldsymbol{a}\right)\right]^{2}\right\}$

Here, $F\left(x_{p}\right)$ is the value of the original function from (4) and (5) evaluated at the point of the design parameters space $\boldsymbol{x}_{p}$, and $P$ is the total number of such plan points; $w_{p}$ is the weight factor that characterises the relative contribution of the information about the original function at the point $\boldsymbol{x}_{p}$. Simple but quite efficient approximations implemented in the method are intrinsically linear (with respect to the tuning parameters) models, namely, linear and multiplicative models:

$\widetilde{F}(\boldsymbol{x})=a_{0}+\sum_{i=1}^{P} x_{i} a_{i}$ and $\tilde{F}(\boldsymbol{x})=a_{0} \prod_{i=1}^{P}\left(x_{i}\right)^{a_{i}}$

More information about the MARS method can be found in the papers of Toropov (1989), Markine (1999), Toropov et al. (1999) and Markine and Toropov (2002).

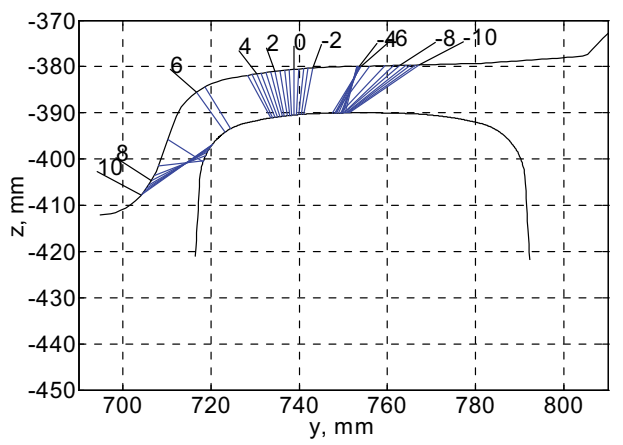

a.

\section{Numerical results}

The wheel profile design procedure described above has been applied to improve the performance of the RET metro trains in Rotterdam (The Netherlands).

\subsection{History}

In 1999, during the RET track renewal, the existing rails with the profile NP46 were replaced by the rails with the profile S49. The profiles looked similar, and therefore no difference in the train performance was expected. However, after the introduction of the new rail profile, the metro trains started to experience severe lateral vibrations. At the same time, a high level of wheel wear after relatively small mileage was observed. In fact, these vibrations were caused by the worn wheels. Immediately after these vibrations had been observed, the wheels had to be replaced (re-profiled) to prevent train derailment. Thus, due to the replacement of the rails, the lifetime of the wheels was reduced from $120,000 \mathrm{~km}$ (with the rails NP46) to $15,000 \mathrm{~km}$ (with the rails $\mathrm{S} 49$ ).

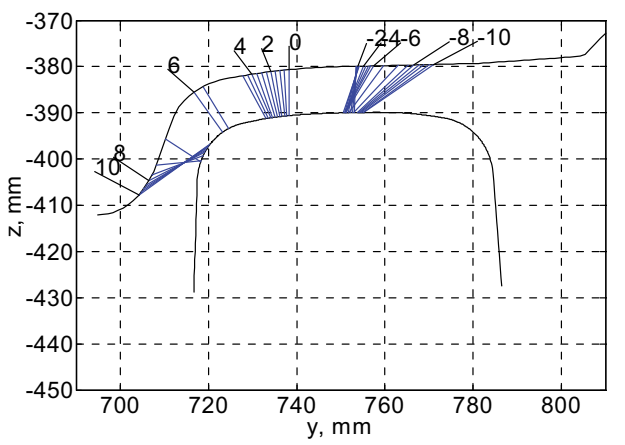

b.

Fig. 9 Contact points of unworn wheel (UIC510) and rails-NP46 a, S49 b 


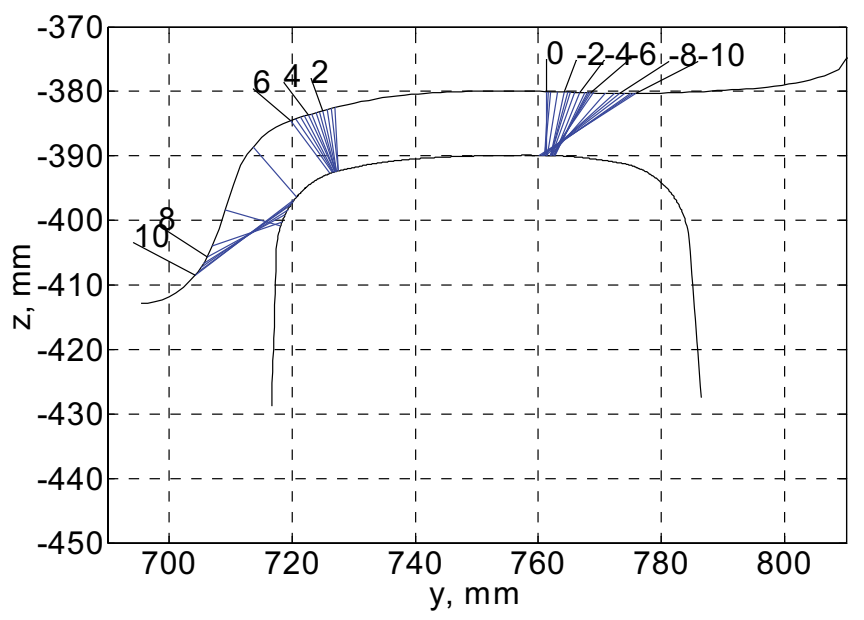

Fig. 10 Contact points of worn (measured) UIC510 wheel and S49 rail

The Railway Engineering Group of TU Delft has been asked to look at the problem and to try to improve the wheel profile with the aim of elimination of the vehicle vibrations, reduction of wheel wear and ultimately increase of the lifetime of the wheels.

\subsection{Analysis of the problem}

The problem has been solved in several steps. First of all, a comparison of the old and new rails has been performed. It should be noted that the S49 rail has the cant of 1:40 in the track to achieve the shape of the NP46 rail, which is used without cant. Even though the rail profiles NP46 and S49 look similar (Fig. 8a), there is a small difference between them which becomes visible only when zooming in the top of the rails as shown in Fig. 8b. Interesting is that such a small difference (order of tenths of a millimetre) in the shape of the rail profile resulted in substantial changes in the dynamic behaviour of the RET vehicles.

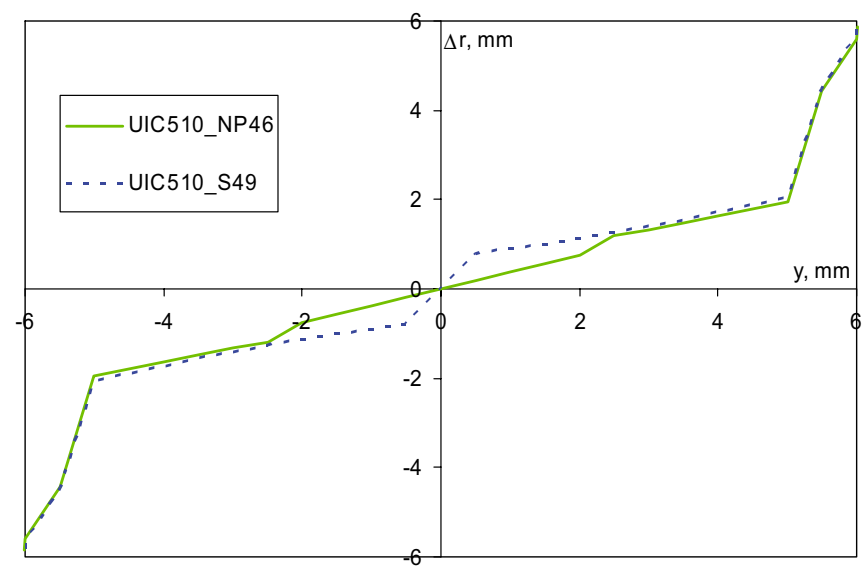

Fig. 11 RRD function: UIC510 wheel on NP46 rail; UIC510 wheel on S49 rail
After that, the wheel/rail contact characteristics of the old and new situations have been investigated. The wheel/rail contact points for the new (unworn) wheel profile UIC510 with the unworn NP46 and S49 rails, respectively, are shown in Fig. 9. In this figure, the lines between the wheel and rail profiles connect the corresponding contact points, which were calculated for each $0.5 \mathrm{~mm}$ of the lateral displacement of the wheelset. Some values of the wheelset lateral displacements are shown above the wheel profile. The coordinate system in this figure is the wheelset coordinate system (Fig. 2) with the origin in the centre of the wheelset in the neutral position. It should be noted that in this figure, the wheel is shifted vertically on $10 \mathrm{~mm}$ upwards from its actual position.

By comparing Fig. 9a, b, one can observe that there is a discontinuity (a big jump) in the position of the contact point on the S49 rail for the $\pm 2 \mathrm{~mm}$ lateral displacements of the wheelset. Because the displacements in this range $( \pm 2 \mathrm{~mm})$ typically correspond to the motion of a wheelset on a straight track (Fig. 1), a lot of such jumps will occur during the vehicle motion. Due to these jumps, a wheel profile wears very rapidly, which ultimately results in very big jumps of the contact point as it is shown in Fig. 10. This figure shows the contact points of the worn (measured) UIC510 wheel profile and the S49 rail. Such big jumps of a contact point were the source of the vibrations observed in the metro trains. On the other hand, the wheel/rail combination UIC510-NP46 has very well-distributed contact points on a straight track as shown in Fig. 9a. As a result, the wear rate of the UIC510 wheels on the NP46 rails was much lower, and therefore the wheel life was relatively long $(120,000 \mathrm{~km})$.

As it was mentioned above, the RRD plays an important role in the vehicle dynamics. Therefore, the RRD functions have been investigated next. The RRD functions of the UIC510 wheel profile on the NP46 and S49 rails are shown in Fig. 11. From this figure, one can observe that for small displacements of a wheelset, the RRD function of the UIC510/S49 wheel/rail combination has much higher inclination as compared to the UIC510/NP46 wheel/rail combination. This also means that the equivalent conicity (2) for

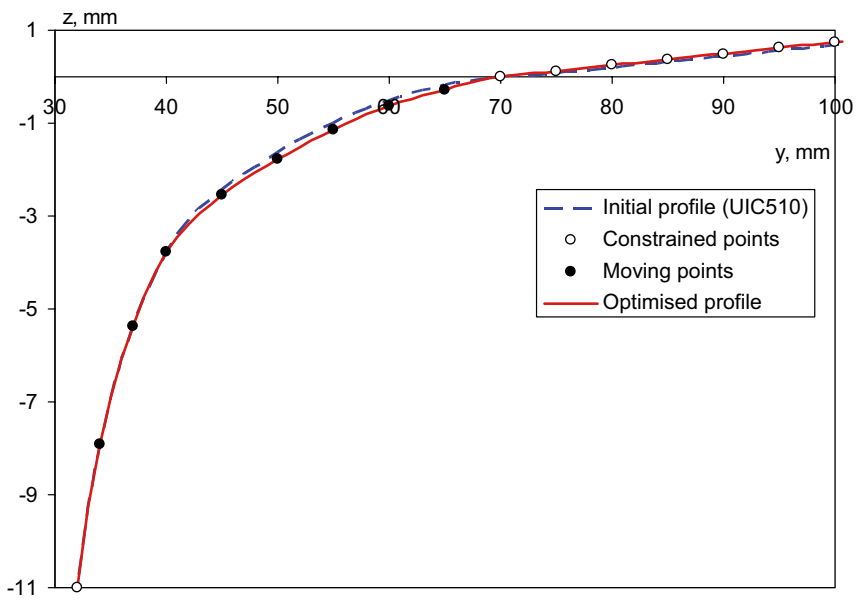

Fig. 12 Initial and optimised wheel profiles 


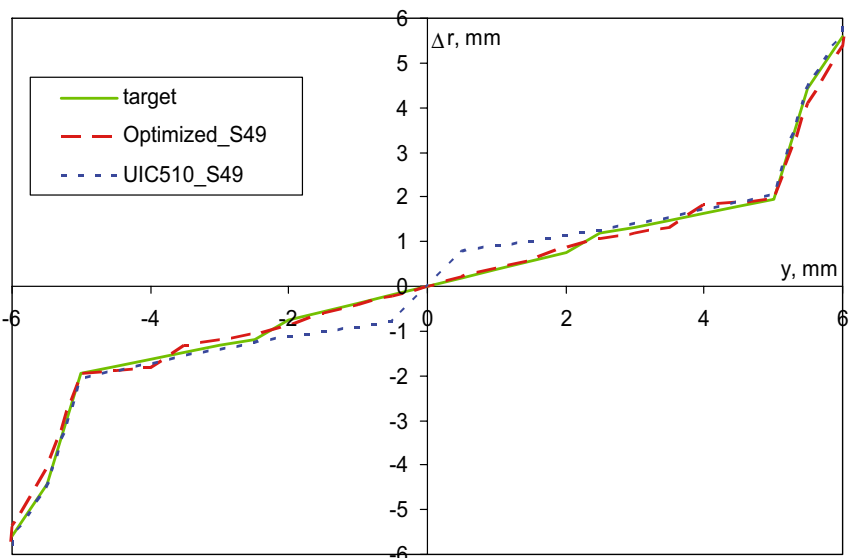

Fig. 13 RRD functions of initial and optimised wheel profiles and target RRD

the S49 rail is higher than the one for the NP46 rail. The high equivalent conicity for small lateral displacements of a wheelset was the reason of the high tread wear, vehicle vibrations and ultimately the relatively short life of the wheels.

\subsection{Design of a wheel profile}

After the source of the vehicle instability problem had been found, the next step was to improve (optimise) the wheel profile. The optimum wheel profile design procedure described earlier in this paper has been used. In this particular case, a practical approach has been used in the definition of the optimum (target) RRD function, namely, the RRD function of the UIC510/NP46 wheel/rail combination (solid line in Fig. 11), for which no vibrations and long lifetime of wheels were observed, has been chosen as the target RRD function. It was expected that the wheels with the optimised profile would have the same lifetime as the UIC510 wheels on the NP46 rails. The multiplicative approximations (14) have been used

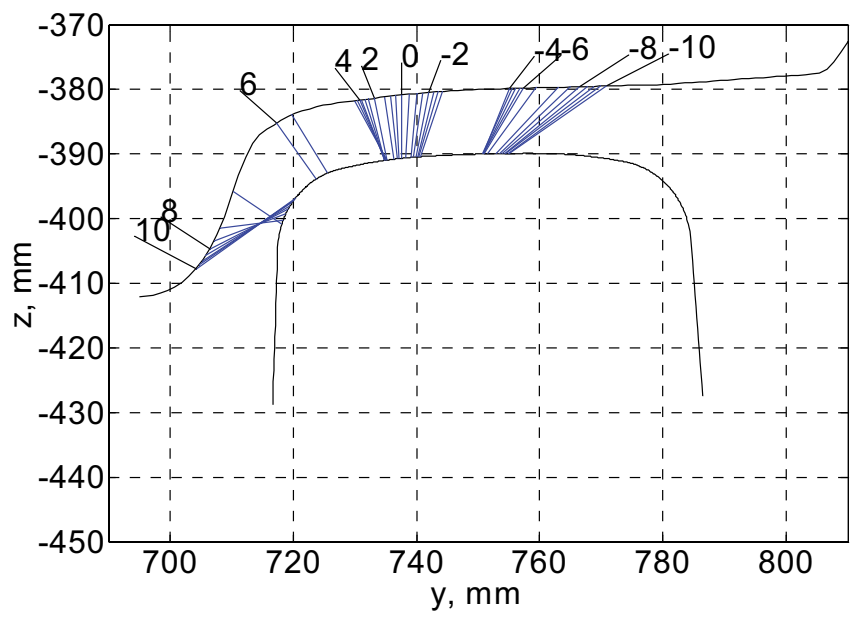

Fig. 14 Contact points of optimised wheel profile and S49 rail
Table 1 Critical velocities of a metro train for various wheel profiles on $\mathrm{S} 49$ rail

\begin{tabular}{ll}
\hline Wheel profile & Critical velocity $\left(V_{c r i t}\right)$ \\
\hline UIC510 worn & $30 \mathrm{~m} / \mathrm{s}$ \\
UIC510 unworn & $50 \mathrm{~m} / \mathrm{s}$ \\
Optimised & $>60 \mathrm{~m} / \mathrm{s}$ \\
\hline
\end{tabular}

in the MARS method to solve the optimisation problem. The results of the optimisation are discussed below.

The shape of the optimum wheel profile is shown in Fig. 12. Even though the changes in the shape are not significantly big, they result in quite substantial changes of the RRD function as it is shown in Fig. 13. From this figure, it also can be seen that the optimised and the target RRD functions are very closed, which means that the MARS method can successfully be applied to such problems. The wheel/rail contact points for the optimised wheel and the S49 rail are shown in Fig. 14. From this figure, it can be observed that the contact points are very well distributed in the range from -4 to $4 \mathrm{~mm}$ of the lateral displacement of a wheelset.

When the optimum profile has been obtained, the dynamic behaviour of the metro vehicle has been analysed. Firstly, the critical velocities of the metro train with the UIC510 and the optimised wheel profiles on the rail S49 have been evaluated. They are collected in Table 1. These velocities have been obtained by performing a series of the dynamic simulations of the metro train with different velocities. From this table, it can be seen that the critical velocity of the metro train has been increased (compare $V_{\text {crit }}=50 \mathrm{~m} / \mathrm{s}$ for unworn UIC510 and $V_{\text {crit }}>60 \mathrm{~m} / \mathrm{s}$ for the optimised wheel profile). The critical velocity for the worn UIC510 profile is extremely low, which explains the vibration problem observed in the metro trains.

After the analysis of the critical velocities, the dynamic simulations of the metro train have been performed to analyse the vehicle stability and wear of the wheels. In these simu-

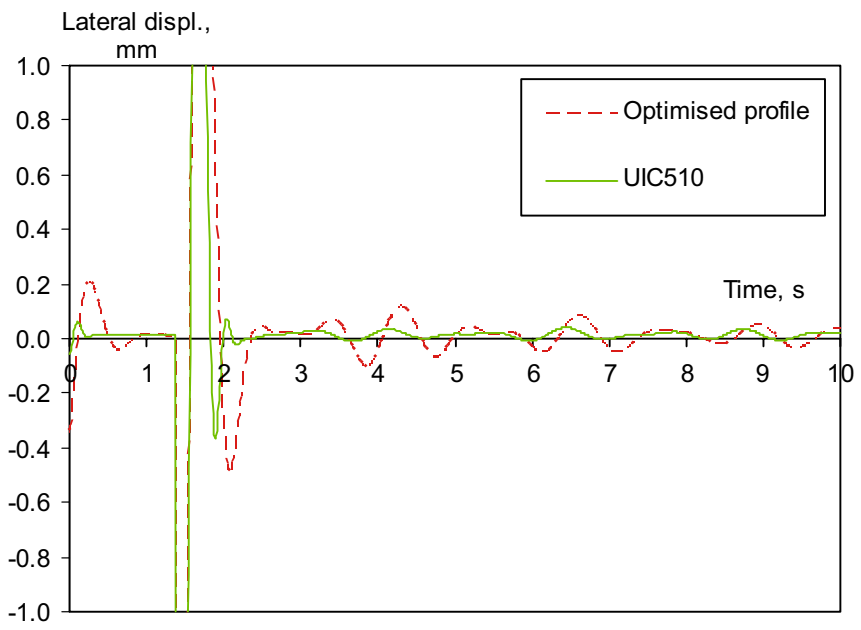

Fig. 15 Lateral displacements of wheelset with optimised and UIC510 wheels on S49 rail, velocity $20 \mathrm{~m} / \mathrm{s}$ 


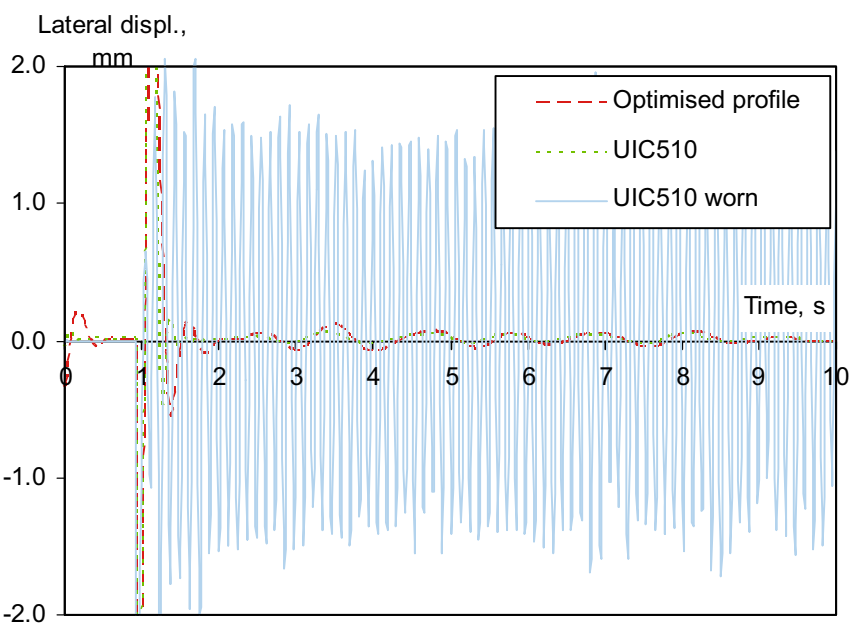

Fig. 16 Lateral displacements of wheelset with optimised, UIC510 and worn UIC510 wheel profiles on S49 rail, velocity $30 \mathrm{~m} / \mathrm{s}$. Worn UIC510 wheel profile is unstable

lations, the metro train was travelling on the straight track, which was described in the previous section, with the operational speed of $20 \mathrm{~m} / \mathrm{s}$. The lateral displacements of its wheelset are shown in Fig. 15. From this figure, it can be seen that the motion of the metro vehicle with the optimised wheel profile is stable at the operational speed of $20 \mathrm{~m} / \mathrm{s}$. From Fig. 15, one can see that the unworn UIC510 wheel profile on the S49 rail delivers stable behaviour of the wheelset at $20 \mathrm{~m} / \mathrm{s}$ as well, which was also observed in practice. For comparison, an example of unstable behaviour of a wheelset is shown in Fig. 16. In this figure, the lateral displacements of the metro train wheelset with the optimised and (worn and unworn) UIC510 wheel profiles travelling on the S49 rails with velocity of $30 \mathrm{~m} / \mathrm{s}$ are shown. The worn UIC510 profile is unstable in this case.

The wear indexes of the wheelset with the optimised and UIC510 wheel profiles are shown in Fig. 17. From this figure,

\section{Wear index, $\mathrm{N}$}

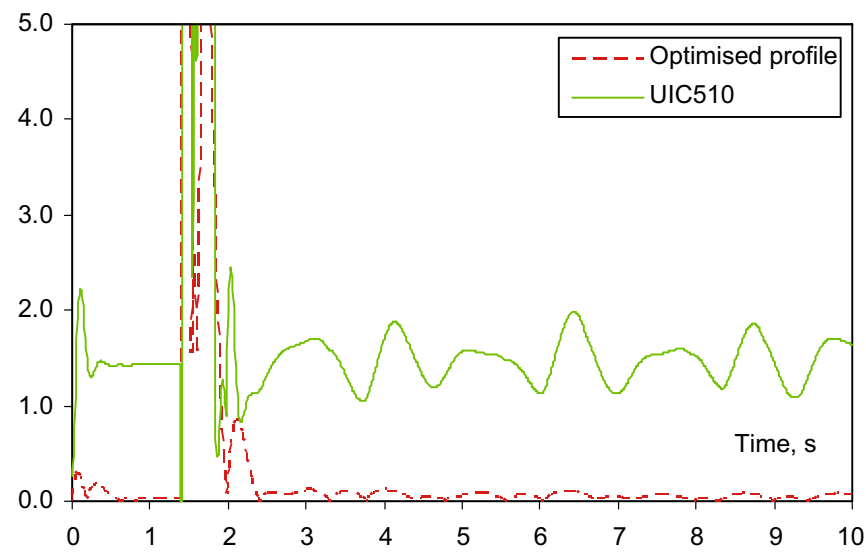

Fig. 17 Wear index of the left front wheel. Optimised and UIC510 wheel profiles on $\mathrm{S} 49$ rail, velocity $20 \mathrm{~m} / \mathrm{s}$

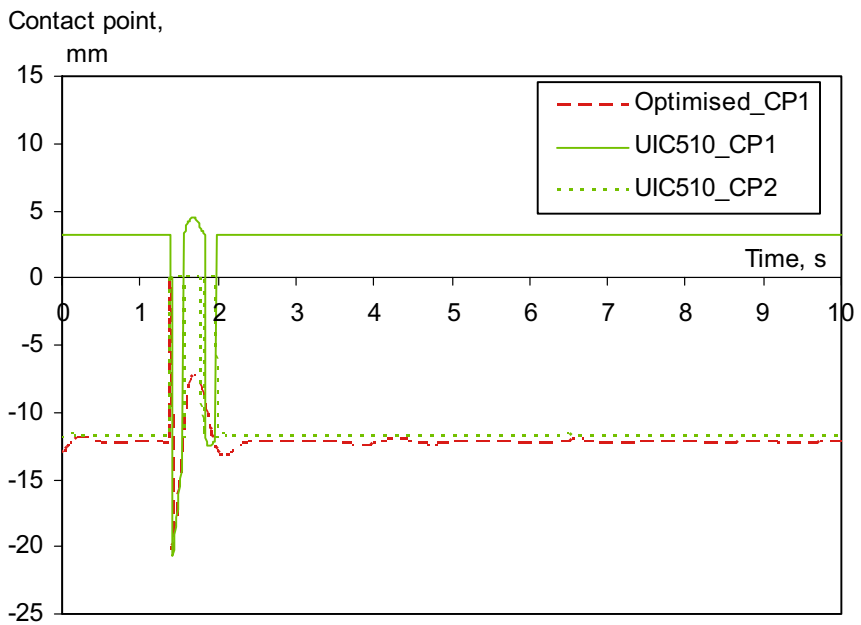

Fig. 18 Position of contact point(s) on the left front wheel. Optimised (single-point contact) and UIC510 (double-point contact) wheel profiles on $\mathrm{S} 49$ rail, velocity $20 \mathrm{~m} / \mathrm{s}$

it can be seen that the wear index for the unworn UIC510/S49 wheel/rail combination is significantly higher than the one calculated for the optimised wheel profile. The explanation can be found in Fig. 18 where the positions of the wheel contact point(s) for these combinations are shown. As it can be seen, the optimised wheel on the S49 rail has single-point contact whilst the unworn UIC510/S49 wheel/rail combination always has double-point contact on the tread part of the wheel. The double-point contact normally results in high wear of wheels. Due to the elimination of the double-point contact, the metro train with the optimised profile should have less wear of the wheels on a straight track and ultimately longer life of the wheels.

The optimised wheel profile was tested by RET. One metro train equipped with the wheels with the optimised profile was running and monitored in the RET network (in 2005-2006). Wear of wheels was measured monthly (after approximately every $10,000 \mathrm{~km}$ ) during the period of 12 months. At the end of the field testing (after approximately $120,000 \mathrm{~km}$ ), wear of the wheels was considerably lower as compared to wear of the UIC510-S49 combination. No oscillations of the vehicle were observed, and the wheels still did not require re-profiling. Thus, due to the implementation of the optimised wheel profile in the RET network, the instability of the metro trains was eliminated, and the life of the wheels (the time between the wheels' re-profiling) was increased from 25,000 to $120,000 \mathrm{~km}$.

\section{Conclusions}

An inverse shape design procedure for railway wheel profiles using numerical optimisation has been presented. The procedure uses the optimality criteria based on the RRD function. According to this procedure, the optimised wheel profile 
is obtained by minimising the difference between the target RRD and the RRD of the designed wheel profile. The MARS method is chosen as a general optimisation technique. This design procedure allows developing wheel profiles with a priori defined contact properties.

The presented procedure has been used for improvement of the wheel profile design for RET metro trains. The target RRD function has been obtained by modifying the RRD function of the existing wheel and rail profiles used in the RET network. The results of the field testing of the optimised wheel profile in the RET network have shown that due to the application of the optimised wheel profile, the instability of the metro trains was eliminated, and the life of the wheels was increased from 25,000 to $120,000 \mathrm{~km}$.

The presented application has demonstrated that the performance of railway vehicles can significantly be improved by improving the wheel/rail contact properties. It was also shown that wear of the wheels can significantly be reduced by eliminating the double-point contact between the wheel and the rail.

\section{References}

ADAMS/Rail (2005) MSC.Software Corporation MSC.ADAMS ${ }^{\circledR}$, http://www.mscsoftware.com

Barthelemy J-FM, Haftka RT (1993) Approximation concept for optimum structural design-a review. Struct Optim 5:129-144

Cooperrider NK, Law EH et al (1976) Analytical and experimental determination of nonlinear wheel/rail constraints. Proceedings of the ASME symposium on railroad equipment dynamics

Dukkipati RV (2000) Vehicle dynamics, CRC Press, Boca Raton, FL. ISBN 0-8493-0976-X

Esveld C (2001) Modern railway track, 2nd edn. MRT-Productions, Zaltbommel. ISBN 90-8004-324-3-3

Kalker JJ (1990) Three-dimensional elastic bodies in rolling contact. Kluwer, Dordrecht. ISBN 0-7923-0712-7

Leary JF, Handal SN, Rajkumar B (1990) Development of freight car wheel profiles - a case study. Proceedings of the third international conference on contact mechanics and wear of rail/wheel systems, Cambridge, U.K., 22-26 July 1990. ISBN 0444-88774-1; also in Wear (1991), vol 144, pp 353-362

Markine VL (1999) Optimization of the dynamic behaviour of mechanical systems. Ph.D. thesis, Shaker Publishing BV, TU Delft. ISBN 90-423-0069-8
Markine VL, Toropov VV (2002) Use of high- and low-fidelity models in approximations for design optimization. Proceedings of the 9th AIAA/USAF/NASA/ISSMO symposium on multidisciplinary analysis and optimization, Atlanta, Georgia, USA, 4th-6th September 2002 (CD proceedings). AIAA Paper 2002-5651

Markine VL, Shevtsov IY, Esveld C (2004) Shape optimisation of railway wheel profile. Proceedings of 21 st international congress of theoretical and applied mechanics, 15-21 August 2004, Warsaw, Poland. (On CD-ROM) ISBN 83-89697-01-1

de Pater AD (1988) The geometrical contact between track and wheelset. Veh Syst Dyn 17:127-140

Persson I, Iwnicki SD (2004) Optimisation of railway profiles using a genetic algorithm. Veh Syst Dyn 41:517-527 (Suppl). ISBN 90265-1972-9

Shen G, Ayasse JB, Choller H, Pratt I (2003) A unique design method for wheel profiles by considering the contact angle function. Proc Inst Mech Eng F J Rail Rapid Transit 217:25-30

Shevtsov IY, Markine VL, Esveld C (2002a) One procedure for optimal design of wheel profile. Proceedings of the IQPC conference on achieving best practice in wheel/rail interface management, Amsterdam, The Netherlands, 31 January-1 February 2002

Shevtsov IY, Markine VL, Esveld C (2002b) Optimization of railway wheel profile using MARS method. Proceedings of the 43rd AIAA/ASME/ASCE/AHS/ASC/ structures, structural dynamics, and material conference, Denver, Colorado, USA, 22-25 April 2002. Paper AIAA 2002-732. ISBN 1-56347-560-X (on CD-ROM)

Shevtsov IY, Markine VL, Esveld C (2003) Optimal design of wheel profile for railway vehicles. Proceedings of the 6th international conference on contact mechanics and wear of rail/wheel systems, Gothenburg, Sweden, 10-13 June 2003, pp 231-236. ISBN 91-6313928-6

Shevtsov IY, Markine VL, Esveld C (2005) Optimal design of wheel profile for railway vehicles. Wear 258:1022-1030. ISSN 0043-1648

Smith RE, Kalousek J (1990) A design methodology for wheel and rail profiles for use on steered railway vehicles. Proceedings of the third international conference on contact mechanics and wear of rail/wheel systems, Cambridge, U.K., 22-26 July 1990. ISBN 0444-88774-1; also in Wear (1991), volume 144, pp 329-342

Toropov VV (1989) Simulation approach to structural optimization. Struct Optim 1:37-46

Toropov VV, Markine VL (1996) The use of simplified numerical models as mid-range approximations. Proceedings of the 6th AIAA/USAF/NASA/ISSMO symposium on multidisciplinary analysis and optimization, Part 2, Bellevue, WA, 4-6 September 1996, pp 952-958. ISBN 1-56347-218-X

Toropov VV, van Keulen F, Markine VL, Alvarez LF (1999) Multipoint approximations based on response surface fitting: a summary of recent developments. In: Toropov VV (ed) Proceedings of the 1st ASMO UK/ISSMO conference on engineering design optimization, Ilkley, West Yorkshire, UK, 8-9 July 1999, pp 371-381. ISBN 0-86176-650-4

Wickens AH (1965) The dynamic stability of railway vehicle wheelsets and bogies having profiled wheels. Int J Solids Struct 1:319-341 